Article

\title{
A Review of In-Situ and Remote Sensing Technologies to Monitor Water and Sanitation Interventions
}

\author{
Luis Andres ${ }^{1, *}$, Kwasi Boateng ${ }^{2}$, Christian Borja-Vega ${ }^{1}$ and Evan Thomas ${ }^{3}$ (iD \\ 1 World Bank Water Global Practice, Washington DC 20433, USA; cborjavega@worldbank.org \\ 2 Portland State University, Portland, OR 97201, USA; kboatengjr@gmail.com \\ 3 Mortenson Center in Engineering for Developing Communities, University of Colorado at Boulder, \\ Boulder, CO 80309, USA; evan.thomas@colorado.edu \\ * Correspondence: Landres@worldbank.org; Tel.: +1-202-473-0819
}

Received: 16 April 2018; Accepted: 25 May 2018; Published: 9 June 2018

\begin{abstract}
The United Nations Sustainable Development Goals (SDGs), announced in September 2015, present a vision of achieving a higher level of human health and well-being worldwide by the year 2030. The SDG targets specific to water and sanitation call for more detailed monitoring and response to understand the coverage and quality of safely managed sources. It is hoped that improved monitoring of water and sanitation interventions will reveal more cost-effective and efficient ways of meeting the SDGs. In this paper, we review the landscape of approaches that can be used to support and improve on the water and sanitation targets SDG 6.1, “By 2030, achieve universal and equitable access to safe and affordable drinking water for all", and SDG 6.2, "By 2030, achieve access to adequate and equitable sanitation and hygiene for all and end open defecation, paying special attention to the needs of women and girls and those in vulnerable situations".
\end{abstract}

Keywords: water and sanitation; monitoring and evaluation; sensors; remote sensing technologies; sustainable development goals

\section{Introduction}

The United Nations Sustainable Development Goals (SDGs) for 2030 were announced with fanfare in September 2015. Updating the Millennium Development Goals (MDGs), the 17 SDGs cover an ambitious range of global topics and include commitments to "End poverty in all its forms everywhere", "Ensure access to affordable, reliable sustainable and modern energy for all," and "revitalize the global partnership for sustainable development" [1].

The new 2030 agenda has water and sanitation at its core, with a dedicated SDG 6 declaring a commitment to "Ensure availability and sustainable management of water and sanitation for all". SDG 6 global targets are universally applicable and aspirational. However, each government must decide how to incorporate them into national planning processes, policies and strategies based on national realities, capacities, levels of development and priorities. Monitoring progress toward this goal will be challenging; current methods of measuring the quality and use of water and sanitation services are either expensive or elusive. For example, a continued reliance on household surveys poses limitations, and such surveys likely overstated progress during the MDG period. The targets and proposed indicators of SDG 6, focused on water, center on "safely managed" services. To be considered "safely managed", a water service must be from an "improved" source (a status defined under the MDGs) on premises, be available as needed, and be free from contamination. A safely managed sanitation service, meanwhile, involves an "improved" sanitation facility that is not shared with other households. Also, human excreta should be (i) treated and disposed of in situ; (ii) stored 
temporarily and then emptied, transported, and treated off-site; or (iii) transported through a sewer with wastewater and then treated off-site [2]. These additional requirements-i.e., those needed to shift from "improved" to "safely managed" water and sanitation services-would cost approximately $\$ 114$ billion per year. Hutton and Vergughese [3] estimate the global capital costs of achieving universal access to Water, Sanitation and Hygiene (WASH) services by 2030 are US\$28.4 billion per year (range: US $\$ 13.8$ to US $\$ 46.7$ billion) from 2015 to 2030 for basic WASH (similar to "improved" standard), and $\$ 114$ billion per year (range: $\$ 74$ to $\$ 166$ billion) for safely managed WASH to meet [3]. Monitoring progress toward them, and associated outcomes, is critical to ensure that available funding is spent wisely over time.

Against this backdrop emergent technologies, methods, and data-sharing platforms are being used to monitor the impacts of water supply, sanitation, and hygiene (WASH) interventions. These may focus on improving service quality or access to service. The improved monitoring of WASH interventions, it is hoped, will reveal increasingly cost-effective and efficient ways to meet SDG 6. New technologies and methods may facilitate the collection and analysis of increasingly complete and impartial data over time-with significant implications for program improvements. For instance, sensor networks are ubiquitous in most developed markets, where they are used to monitor industrial processes, track products, and organize information services. More recently, many organizations have begun leveraging sensors for supply chain management, remote monitoring, and consumer product testing.

Data underpin the governance elements of accountability and transparency. They enable progress to be monitored and service providers, governments and development partners to be held accountable. Many countries lack the financial, institutional and human resources needed to acquire and analyze data to support governance. Less than half of US Member States have comparable data available on progress towards meeting each of the global SDG 6 targets.

Stakeholders have no factual data for assessing and challenging, when needed, incorrect or biased positions. Reliable, consistent and, whenever possible, disaggregated data are essential to stimulate political commitment, inform policy-making and decision-making, and trigger well-placed investments. Political commitment to transparency that includes efforts related to accessibility and sharing of data is essential for data acquisition and monitoring. Increased utilization of the latest Earth observations, citizen science and private sector data should be incorporated into data monitoring systems at all levels to complement existing data-collection efforts.

In this paper, we review the landscape of technologies, methods, and approaches that can support and improve on the water and sanitation indicators proposed for SDG targets 6.1 ("By 2030, achieve universal and equitable access to safe and affordable drinking water for all") and 6.2 ("By 2030, achieve access to adequate and equitable sanitation and hygiene for all and end open defecation, paying special attention to the needs of women and girls and those in vulnerable situations").

In some cases, technologies and methods are proven and readily available. In other cases, emergent technologies and approaches hold promise but require further field evaluation and cost reductions. In any case, considering which emergent technologies will most effectively monitor progress toward the SDGs is of critical importance as stakeholders seek to identify which kinds of policies and programs are working well, and which require corrective measures.

Efforts to measure the adoption of and compliance with key water and sanitation technologies—-such as latrines, water pumps, and water filters-have often relied on surveys and field observations. Despite what some consider to be the gold standard in WASH monitoring and evaluation, structured observation still has substantial limitations. For one, implementation is resource intensive, requiring time, labor, and funds. Quality enumerators and comprehensive training are required to limit bias due to respondent reactivity and observer differences. This is especially important when the measurement is fairly subjective-inclusive of not only self-reported behaviors and proxy indicators, but also those dependent on observer interpretation - as this has been shown to bias effect estimates [4]. Secondly, the length of time the observer is in the household may be inconvenient for study participants 
and may change their routine [5]. Thirdly, similar to survey reactivity, the act of repeated observation has been shown to augment the behavior of interest, known as the Hawthorne effect [6]. The presence of a human observer outside of a latrine modifies the usual behavior of households, increasing the use of the latrine significantly [7]. Similarly, one study showed soap use after crucial events is higher in the first 90 min of observation than in the following three and a half hours [5]. Additionally, households with higher social status show more reactivity to the observer, meaning the exercise may capture information about the population differentially [8]. Finally, direct observation has been shown to have low repeatability in individual monitoring and may only be appropriate to measure behavior at a population level or after repeated observations [9], although the latter has the potential to produce a Hawthorne effect as well.

While the new SDG indicators for WASH build on established indicators, thereby providing some continuity that will help ongoing monitoring efforts, they also move beyond them. This places considerable demand on national household surveys to develop new data collection methodologies.

Emergent technologies-including water meters, water pump sensors, and latrine motion detectors-can improve the objectivity of data collection. Satellite-based remote sensors linked to the Internet of Things can be aligned with smartphone-based surveys and online "big data" tools. In addition, high-resolution smart water meters and advanced data analytics allow for a new era of using the continuous "big data" generated by these meter fleets to create an intelligent system for urban water management. These technologies can also provide more transparency and accountability means from governments when delivering these types of services to the population. Governments fulfil this responsibility by using evidence and updated data for planning, regulating, financing, budgeting and monitoring services and providing information about them. Government decisions depend on the extent to which information is reliable and monitored on a timely basis, to also underpin the service delivery arrangement. This involves, in addition, increasing accountability of the operating environment for the providers, delegation, contracting, or licensing of operations, so that providers deliver services to the users.

Remote sensing technologies may be of critical importance where limited information is available. For example, the spatial data behind remote sensing imagery can be used to feed a robust spatial econometric model to estimate the severity of infrastructure deprivation in the slums and shantytowns of a given city, where surveys typically are not conducted due to high data collection costs. In situ instruments vary from flowmeters and water quality sensors to motion detectors installed in latrines. These sensor technologies can be used both operationally or within a sampling frame. Collecting data via cellular phones, with online analytics and dissemination, is an increasingly common feature of water and sanitation programs.

\section{Sensing WASH}

Remote monitoring, via satellite assets and distributed in situ sensors, may help meet many of the challenges of information asymmetry and data gaps in developing countries, including unreliable (and time-consuming) survey data and reliance on spot checks to assess accurate reporting and performance. Remote data can be used to understand programmatic, social, economic, and seasonal changes that may influence the quality of a program. For instance, satellite technology has allowed measurements on a global scale over long time periods, and is now proving useful in coastal waters, estuaries, lakes, and reservoirs, which are relevant to water quality managers. Additionally, the behavioral patterns of users can be studied to better understand how and when water and energy technologies are being used. Further, the information can assist the policy design and implementation of efforts to expand coverage, by providing comprehensive evidence of how best to prioritize and sequence interventions, and by estimating the cost-effectiveness and efficacy of upgrading to "safely managed" services.

In this paper, we review the use of remote sensing and local sensors for water and sanitation monitoring. The term "remote sensing" usually describes the collection of data by satellites. In most 
cases, "remote" refers to spectral imagery collected by cameras and other spectral instruments across a broad range of wavelengths. For observing the earth, satellites take spectral data reflecting from the atmosphere and the Earth's surface. Interpretation of these data (often represented as imagery) requires an understanding of the spectral data and physical properties of the Earth and its atmosphere. It also often requires calibration against data collected on the Earth's surface or in the atmosphere directly-data from sensors that are in situ, rather than remote. Modern optical sensors can capture both qualitative imagery and quantitative information, by using a range of different wavebands of light. This means the data is often in a familiar "photographic" form, showing the Earth from the perspective of space. However, it can also contain additional information that helps researchers and decision makers better understand the dynamics of a specific area and variables that play a key role in determining certain trends. Different wavelengths of light filtered through the imagery can, for instance, identify land boundaries, land classifications, atmospheric water vapor, industrial soil pollution, and sediments.

In situ instrumentation technologies, ranging from flowmeters and water quality sensors to motion detectors installed in latrines to monitor usage or adoption, can complement such information. These sensor technologies can be used both operationally or within a sampling frame to be combined with other dimensions of information. Data can be logged locally for manual retrieval or transmitted over short range to nearby enumerators, or to remote operators and researchers over Wi-Fi, cellular, and satellite networks. Some instrumentation is in common use, while other technologies are emerging. However, given the remote and power-constrained environments involved, and the high degree of variability in the characteristics of fixed infrastructure, including in age, materials, quality, servicing, and functionality, any electronic-sensor-based solution would have to either be bespoke engineering, or compensate for these complexities through analytics. For example, a conventional flowmeter designed for a rural borehole water distribution scheme would have to address pipe diameter, material, pressure, depth, thread type, and other characteristics that require custom engineering and plumbing. Instead, a nonintrusive ultrasonic flowmeter may be more easily adapted for a variety of water schemes.

\subsection{Satellite Remote Sensing}

Remote sensing capabilities and techniques are well suited for the monitoring of regional-scale precipitation, water budgets, soil moisture, and some measures of water quality. Other possible satellite imagery and remote sensing applications include catchment characterization, water quality monitoring, soil moisture assessment, water extent and level monitoring, irrigation service monitoring, urban and agricultural water demand modeling, evapotranspiration estimation, ground water management, hydrological modeling, and flood mapping/forecasting. Various satellite data products have been leveraged to aid water and sanitation programs. Key examples include:

- The Sentinel-1 Program for water management in low-income countries was part of a large research initiative that explored the performance and opportunities provided by the European satellite Sentinel-1 for water resource management applications. The satellite data was used to estimate water volumes retained by small reservoirs to assess the feasibility of small water supply systems [10].

- In particular, LandSat 8 data allow for a calculation of the Normalized Differential Vegetation Index (NDVI). The LandSat 8 NDVI allows an estimation of land surface emissivity [11] and land cover classification [12], as well as surface temperature. These measures can be used by remote sensing experts to allow a planning-level estimation of watershed health across a broad region. Additionally, land use classification can identify the vegetation and population densities of rural versus urban, built environments.

- SERVIR, a cooperative effort of NASA and the United States Agency for International Development (USAID), "works in partnership with leading regional organizations world-wide to help developing countries use information provided by Earth observing satellites and geospatial technologies for managing climate risks and land use" [13]. With three regional offices, SERVIR 
has been able to partner with remote sensing experts and national decision-making bodies. Among other activities, SERVIR focuses on monitoring water bodies to observe effects from "human activities, climate change, and other environmental phenomena". It takes advantage of LandSat, ASTER, MODIS, and other satellite assets to monitor water quality and changes. Specifically, SERVIR is developing rainfall and runoff models to study the availability and quality (as sediment or total dissolved solids) of surface water over the next several decades.

- Using the Tropic Rainfall Measuring Mission (TRMM) data, the Nile Basin Initiative in partnership with NASA provides flood forecasts and water balance estimates for the Eastern Nile Basin. Similarly, the Surface Hydrology Group at Princeton University developed the Africa Drought Monitor and provides maps of rainfall, temperature, and other hydrologic variables [14].

- The USAID Famine Early Warning System Network (FEWS) monitors rainfall and crop production with satellite assets and combines these data with socioeconomic insights to identify population groups that may be vulnerable to food insecurity [15].

- NASA's Terra satellite includes two instruments that have been leveraged for watershed monitoring. The Moderate Resolution Imaging Spectroradiometer (MODIS) and the Multiangle Imaging Spectroradiometer (MISR) satellite assets can be used to determine aerosol optical depth, land surface temperature, enhanced vegetation index, and middle infrared reflectance. Some of these data can be used to assess water quality parameters including chlorophyll, cyanobacterial pigments, colored dissolved organic matter, and suspended matter in water bodies on a large scale [15].

- In Nigeria, the World Bank recently used geographic information system (GIS) mapping techniques to compare household survey data against MODIS land use estimates to generate spatial distribution estimates of water and sanitation indicators, including service access [16].

- In Dhaka, Bangladesh, the World Bank and other partners (University of Massachusetts Boston, and Earth Observation for Sustainable Development/GiSat) used high-resolution imagery in slums to correlate satellite imagery to public service availability [17].

- The Inter-American Development Bank (IDB) developed the Hydro-BID platform to assist countries in Latin America and the Caribbean with water management through the mapping and tracking of over 230,000 water catchment areas. The Hydro-BID platform is leveraged by government agencies and water utilities for regional water management and infrastructure planning [18].

\subsection{Unmanned Aerial Vehicles}

Increasingly, unmanned aerial vehicles (UAVs, or drones), are being used by researchers to map watersheds. Structure from Motion (SfM) three-dimensional point clouds can be derived from digital images collected on board UAVs, similar to the type of data produced by Light Detection and Ranging (LiDAR) technology [19]. The technological development of UAVs and their ability to derive high-resolution 3D information (and with much lower operational and up-front costs than manned airborne platforms) and satellite imaging has made UAV image acquisition appealing in several applications. Research applications utilizing UAVs for environmental monitoring, management, and evaluation have been increasingly explored in the past 10 years. The use of UAVs for data collection in the monitoring of natural resources [20,21], biomass [22], forests [23-25], and vegetation [26,27] have been found to have significant impacts on the temporal and spatial resolution of data at a more cost-effective price than traditional monitoring practices.

\subsection{Water Quality Sensors}

A variety of water quality sensors are commonly used for both in situ continuous monitoring as well as sample-based testing. Off-the-shelf sensors are readily available to measure $\mathrm{pH}$, dissolved oxygen (DO), conductivity (often used to measure salinity), turbidity, temperature, chlorine, various 
dissolved ions (including fluoride, ammonia, silver, nitrate, and nitrite that are often byproducts of fertilizers), and total organic carbon (TOC). A complete review of these products and applications are outside the scope of this report.

Instead, several more recent sensor applications, directly relevant to monitoring SDG targets 6.1 and 6.2, are highlighted. In particular, water quality parameters may be estimated cost-effectively using remote sensing techniques and technologies. Water quality assessments include the identification of colored dissolved organic matter (CDOM), Secchi disk depth (SDD), turbidity, total suspended sediments (TSS), water temperature (WT), total phosphorus (TP), sea surface salinity (SSS), dissolved oxygen (DO), biochemical oxygen demand (BOD), and chemical oxygen demand (COD). With advances in space science, computer applications, and computational methods over recent decades, remote sensing techniques have become useful tools to achieve this goal.

\subsection{Microbial Sensors}

Akvo Caddisfly builds on the smartphone-based application Akvo Flow, a survey-based application similar to previously described smartphone and dashboard programs [28]. However, Akvo Caddisfly integrates pocket-sized water-testing devices directly to the smartphone, whereby results do not need to be entered by the user but are automatically measured and recorded. Currently, Akvo Caddisfly can measure several water-quality parameters. Fluoride is measured using a test chamber through which a reagent is added to the sample and a photo is taken to analyze the concentration based on the color generated by the sample and reagent mixture. Salinity is measured based on an electrical conductivity sensor attached directly to the phone via a USB cable. Additionally, any color-based test strip can be analyzed by Akvo Caddisfly by corresponding it to the color calibration card, thereby limiting the subjectivity of the test.

A class of optical fluorimeters may be used as real-time in situ fecal contamination detectors. These sensors detect tryptophan-like fluorescence (TLF) associated with the presence of microbial contamination. In recent years, several laboratory-grade products have been validated in the field as predictive of E. coli $[29,30]$. However, these instruments require frequent cleaning, are not intended for long-term autonomous operation, and cost $\$ 7500$ or more.

These state-of-the-art fluorimeter solutions are not designed for in situ long-term use. This is primarily because biofouling and baseline drift attenuate and confuse the signal [31]. At present, there are no viable in-situ electronic sensors for monitoring the microbial contamination of drinking water. The closest products feasible for this application are spectroscopy-based sensors to detect TLF from E. coli. The United Nations Children's Fund (UNICEF) has recently published a Target Product Profile detailing a market need for improved sensor systems for the microbial contamination of drinking water [32].

\subsection{Chemical Sensors}

For municipalities, water quality detection and monitoring systems have to be reliable, autonomous, and flexible. For example, in some countries fluoride is a priority chemical constituent to be monitored, since its consumption at early ages can induce fluorosis, which affects the teeth and bones of children. There are several commercially available sensor products for fluoride monitoring including the Hach Sension+ Fluoride Ion Electrode, and various in-line sensors for larger-scale water distribution systems such as the Rosemount Fluoride Monitoring System [33], and the ATI Fluoride Monitor [34]. These technologies can even sense moderate levels of chronic exposure to fluoride concentrations (above 1.5 milligrams per liter (mg/liter) of water-i.e., the World Health Organization (WHO) guideline value for fluoride in water), which can help prevent risk of disease.

In Bangladesh, where arsenic is a widespread contaminant impacting health (causing the deaths of an estimated 43,000 people each year), researchers and practitioners are developing and testing biosensors that can be rapidly deployed to indicate arsenic contamination in drinking water [35]. 
Similarly, there are several off-the-shelf sensor-based technologies for monitoring arsenic contamination, a priority chemical for SDG 6 monitoring. These include the Palintest Digital Arsenic Test Kit [36] and the IPI Digital Arsenic Detector [37]. However, there remains a need for additional technology development in this area, as was highlighted by the recent United States Environmental Protection Agency (US EPA) Arsenic Sensor Prize Competition [38].

\subsection{Water Service Delivery and Use}

Water flowmeters are commonly used to measure service delivery and enable billing for the municipal water supply of many cities. These meters vary in functionality and form, from mechanical meters that are manually read to remotely reporting sensors. A challenge facing water service providers and customers is so-called non-revenue water (NRW) - that is, water supply lost in system leaks or used by people but not paid for. In developing countries, leaks alone have been estimated to cost more than $\$ 40$ billion per year, with a loss of 45 billion liters of water-enough water to serve 200 million people. A further 30 billion liters is lost to poor metering or billing processes. This negative feedback loop diminishes the capacity of utilities to deliver reliable, safe water, and the willingness of customers to pay for this service.

Improved water meters and processes can reduce this NRW gap, and improve the quality of water service delivery-and consumers' faith in it. In India, NextDrop [39] technologies have combined remotely reporting water meters with periodic water quality tests to reduce water consumption, leaks, and service outages.

Also, in South Asia (India), the social enterprise Piramal Sarvajal [40] developed a reverse osmosis water treatment tool, "ATM", that continuously monitors and remotely reports on system functionality and water quality. Internet-connected sensors-including smart flow and pressure meters, water quality sensors, smart water consumption meters, and leakage-monitoring sensors-identify flaws and deficiencies in water distribution networks.

In Sub-Saharan Africa, around 1 million hand pumps supply water to over 200 million rural water users across the continent, yet as many as one-third of all such pumps are not working at any given time, with $30 \%-70 \%$ broken within two years of installation. In 2013, Oxford University set up a trial of mobile-enabled technologies for 66 "smart" hand pumps. The study demonstrated that hand pumps with cellular-network-enabled sensors, and that received a repair during the trial, had far less downtime: 2.6 days versus an average of 27 days. Also, participating communities were three times more willing to pay for pump services than they had been before the installation of the sensors [41].

Building on this work, in 2014, Portland State University, Living Water International and SweetSense Inc. installed 181 cellular-enabled water pump use sensors in three provinces of Rwanda. The nominal maintenance model was compared against a "best practice" circuit rider model wherein dedicated staff and supplies were assigned to pump groups, and an "ambulance" service model wherein sensor data triggered a service dispatch. In only the ambulance model were the sensor data available to the implementer and used to dispatch technicians. This longitudinal study demonstrated that a sensor-based service model for hand pumps in rural Rwanda was associated with substantial reductions in the time interval between breakdown and repair when compared with both the nominal service model and a "best-practice" circuit rider approach. Similarly, there were significant, and parallel, differences in the amount of time that pumps in each service model were functional. At the baseline, $56 \%$ of the implementer's pumps were functional, with a mean reported nonfunctional period of approximately 214 days. In the study period, the nominal maintenance group's median time gap between breakdown and successful repair was approximately 152 days, with a mean per-pump functionality of about $68 \%$. In the circuit rider group and the ambulance service group, this time gap was nearly 57 days and 21 days, with a per-pump functionality mean of nearly $73 \%$ and $91 \%$, respectively. 
Smart metering, an approach first matured in the power industry, allows a water utility to obtain meter readings on demand without requiring manual meter readers. A recent IDB report summarized the opportunities and limitations of smart water meters [42].

In two recently funded USAID programs in Ethiopia [43] and Kenya [44], satellite-network-connected sensors were installed on remote, electrically powered boreholes to monitor functionality and water service delivery to thousands of customers. It was hoped that the resulting data would inform decisions regarding when to dispatch technicians, order repair supplies, and initiate other responses as needed.

\subsection{Household Water Use}

Several products are available commercially to monitor residual chlorine, an indication of water treatment practices and water safety. These include pocket colorimeters from Hach, Analyticon DKK-TOA, Omega, and other vendors.

The use of household water filters has also been measured in some studies. In Rwanda, researchers distributed household water filters and collected data on product compliance using: (1) monthly surveys and direct observations by community health workers and environmental health officers; and (2) sensor-equipped filters. The adoption rate interpreted by the sensors varied from the reporting: $96.5 \%$ of households reported using the intervention filter regularly, while the sensors interpreted no more than $90.2 \%$. Also, the sensor-collected data estimated approximately $36 \%$ less water volume per day than survey estimates [45].

Expanding on this study, a cluster randomized controlled trial was later conducted in Rwanda among 170 households (70 blinded to the presence of the sensor, 100 open) to test whether awareness of an electronic monitor would result in a difference in weekly use of household water filters and improved cookstoves over a four-week surveillance period. A $63 \%$ increase in the number of uses of the water filter per week between the groups was observed in week 1 (an average of 4.4 times in the open group, and 2.83 times in the blind group), declining in week 4 to an insignificant $55 \%$ difference (of 2.82 uses in the open and 1.93 in the blind). Use of the water filters decreased in both groups over the four-week installation periods. This study suggests behavioral monitoring should attempt to account for reactivity to awareness of electronic monitors that persists for weeks or more [46].

\subsection{Sanitation}

Several studies have used the Passive Latrine Use Monitor (PLUM) first developed at the University of California at Berkeley and commercialized by SweetSense Inc. The sensor uses a simple passive infrared motion detector to identify warm-bodied movement in a latrine stall. These binary movement data are recorded at three-second intervals, and either logged locally on the sensor device or transmitted by cellular or satellite data networks for online processing. Structured observation-validated algorithms process these movement data into discrete use estimates. These estimates have been shown to be more reliable in individual households than in community latrines where lines may form. In some cases, the sensors include radio-frequency identification (RFID) readers to enable latrine-servicing requests, as demonstrated by Sanergy Inc. in Nairobi, Kenya. Hence, sensing systems can be designed to collect data in a cost-efficient manner, through careful maintenance and reuse (even if the cost of individual sensors is significant).

In one recent study in Bangladesh, these sensors demonstrated a significant overreporting of latrine utilization. Across 1207 households randomly selected from 52 village committees in Bangladesh, the mean four-day self-reported latrine use was 32.8 uses, while sensor analysis suggested 21.7 uses on average, indicating a more than $50 \%$ exaggeration of latrine use. At the low end of the regression model, the intercept suggests that many households report using latrines when in fact no use is detected [47].

Similarly, in a recent cross-sectional study among 292 households in 25 villages in India, these sensors were installed for two weeks and compared with household surveys. The mean reported 
daily use was nearly twice that of the sensor-recorded use, with moderate agreement between daily reported use over the previous $48 \mathrm{~h}$ [48].

Remote sensing technologies can also be applied to larger sanitation infrastructure networks. For instance, some utilities are using remote sensing to optimize routes for meter collection data. The remote sensing information is helping large and complex sanitation systems to identify spatially intelligent routes for infrastructure expansion in densely populated areas, consequently reducing money and time based on spatially integrated information.

\subsection{Handwashing}

Sensors can also provide an objective and nonobtrusive characterization of handwashing behavior. For example, organizations in the water and sanitation sector have used accelerometers and motion sensors to monitor household water consumption, community adoption of latrines, and the use of handwashing stations [49].

SmartSoap, developed by Unilever, is an ordinary looking bar of soap with an embedded accelerometer that measures motion on three axes, allowing the detection of use. On its own, SmartSoap can provide an accurate count of the number of times the soap bar is used each day. Through combining SmartSoap data with data from a motion sensor placed on the vessel holding water for anal cleansing, Biran et al. [50] were able to detect handwashing events after defecation. Although overall soap use increased, they found that there was no increase in the number of soap uses following defecation.

Similarly, Mercy Corps used motion-detector-based latrine sensors combined with water-flow sensors to monitor the prevalence of handwashing after latrine use. They found that water use after latrine use was very low $(<10 \%)$ in all but one district, which registered almost $40 \%$ use of water after latrine use. They also found that self-reported use of a latrine and handwashing after using a latrine were much greater (up to 4 times and 25 times, respectively) than that detected by the sensors [45].

\section{Discussion}

A key challenge in the WASH sector is that of bringing together numerous stakeholders spanning different sectors to consider a multitude of data sets. There is a clear need to ensure the supply of high-quality information and to improve analytical capacity by filling key gaps in WASH data and strengthening data integration across multiple sectors. Today, big data applications stand a chance of helping governments in emerging economies address the myriad social and economic issues that continue to plague these regions. Opening up access to data and improving the transparency of WASH projects will empower consumers to have more fruitful interactions with stakeholders. Collaborative dialogue and efficient tools are sure to provide consumers and governments a way to address social and economic problems [51]. For instance, development organizations are working in partnerships with governments of emerging economies to precisely use big data in producing official statistics, official indicators and report more accurately on SDG targets, including those pertaining to water [52]. The WASH sector, among others, serves as an important source of business value that cuts across multiple industries. This is because WASH sector initiatives, apart from improving the lives of millions, also increase value in manufacturing operations, supply chains, and workforce development, among others. It is important that stakeholders get a clear view of results, if the world's numerous WASH initiatives are to succeed and scale up in the various places they have been deployed. A concerted effort on the part of relevant stakeholders will be required if the full potential of big data is to be realized in global development efforts. For instance, governments need to get involved in regular data collection efforts that are simply inaccessible or extremely difficult for private companies to undertake. These may include but are not limited to censuses, surveys, and other regular sources of information that cover vast areas and large numbers of the general populace. Once these open data initiatives are made available to organizations engaged in big data analysis in global development, those organizations can further tease out any new insights. This requires a shift in paradigms towards the use and capabilities of big data for developing economies. Recent discussion among international 
development organizations suggests moving away from seeing big data in isolation, but to rather focus on the "ecosystem" of big data. According to this concept, big data is not just data, no matter how big or different it is considered to be; big data is first and foremost 'about' the analytics, the tools and methods that are used to yield insights, the frameworks, standards, stakeholders involved and then, knowledge. Moreover, citizen involvement is also crucial if big data initiatives in global development are to succeed. Many citizens are getting involved in monitoring WASH services, and their active participation will go a long way toward opening up interpretations of collected data-and relevant decision making. The pervasiveness of mobile usage in most developing regions can be leveraged in this respect. Access to data empowers citizens to make choices concerning WASH service providers and fiscal decisions-as when presented with the ability to, for example, track their spending on WASH services. The efforts of governments, development organizations, and the private sector have to be properly coordinated to realize potential benefits. Government policies and legal frameworks should adequately protect individuals and corporate entities alike, while development organizations need to constantly demonstrate the public good that big data seeks to offer.

\section{Ways Forward}

Given the myriad of monitoring and evaluation methods, each having their own advantages and limitations, it is often beneficial to leverage more than one method to get a more complete picture of water and sanitation behavior. Combined methodologies reinforce the advantages, while addressing the limitations, of each of the monitoring technique that they are comprised of. Surveys, ethnographies, and direct observation provide context for electronic sensor readings that remain objective despite interim analyses. Sensors or spot checks may capture household characteristics, but surveys, and ideally, structured observations, are used to inform individual behavior, which further refines the algorithm or index for streamlined analysis during subsequent monitoring periods. Overall, combining methodologies promises to provide a comprehensive and instructive depiction of WASH usage.

Some of the technologies and methods presented in this paper are well established, while others hold promise but require extensive field testing and validation, commercialization, and scaling. As such, we have not attempted to directly compare costs. Also, applications vary widely, and it is beyond the scope of our study to compare the relative value or reliability of different methods. Instead, we present a menu of options for policy makers, program implementers, and auditors to consider when designing impact measurement efforts.

Author Contributions: Senior authorship is not assigned.

Conflicts of Interest: This paper builds on a previous publication by the authors published at the World Bank [53]. The World Bank granted to Water a worldwide, non-exclusive license to publish its copyrighted material entitled. Author Thomas is a co-owner of SweetSense Inc., a company referenced in this work. The findings, interpretations, and conclusions expressed in this paper are entirely those of the authors. They do not necessarily represent the view of the World Bank, its executive directors, or the countries they represent. The findings, interpretations, and any remaining errors in this paper are entirely those of the authors.

\section{References}

1. WHO/UNICEF (Joint Monitoring Programme for Water and Sanitation). Progress on Drinking Water, Sanitation, and Hygiene: 2017 Update and SDG Baselines; WHO and UNICEF: Geneva, Switzerland, 2017.

2. World Health Organization (WHO)/UNICEF (Joint Monitoring Programme for Water and Sanitation). Methodological Note: Proposed Indicator Framework for Monitoring SDG Targets on Drinking-Water, Sanitation, Hygiene and Wastewater; WHO and UNICEF: Geneva, Switzerland, 2015.

3. Hutton, G.; Verughese, M. The Costs of Meeting the 2030 sustainable Development Goal Targets on Drinking Water, Sanitation, and Hygiene. In Water and Sanitation Program; World Bank: Washington, DC, USA, 2016.

4. Wood, L.; Egger, M.; Gluud, L.L.; Schulz, K.F.; Jüni, P.; Altman, D.G.; Gluud, C.; Martin, R.M.; Wood, A.J.G.; Sterne, J.A.C. Empirical evidence of bias in treatment effect estimates in controlled trials with different interventions and outcomes: Meta-Epidemiological study. British Med. J. 2008, 336, 601-605. [CrossRef] [PubMed] 
5. Halder, A.K.; Molyneaux, J.W.; Luby, S.P.; Ram, P.K. Impact of duration of structured observations on measurement of handwashing behavior at critical times. BMC Public Health 2013, 13, 705. [CrossRef] [PubMed]

6. Zwane, A.P.; Zinman, J.; Dusen, E.V.; Pariente, W.; Null, C.; Miguel, E.; Kremer, M.; Karlan, D.S.; Hornbeck, R.; Giné, X.; et al. Being surveyed can change later behavior and related parameter estimates. Proc. Natl. Acad. Sci. U.S.A. 2011, 108, 1821-1826. [CrossRef] [PubMed]

7. Clasen, T.; Fabini, D.; Boisson, S.; Taneja, J.; Song, J.; Aichinger, E.; Bui, A.; Dadashi, S.; Schmidt, W.P.; Burt, Z.; et al. Making sanitation count: Developing and testing a device for assessing latrine use in Low-Income settings. Environ. Sci. Technol. 2012, 46, 3295-3303. [CrossRef] [PubMed]

8. Ram, P.K.; Halder, A.K.; Granger, S.P.; Jones, T.; Hall, P.; Hitchcock, D.; Wright, R.; Nygren, B.; Islam, M.S.; Molyneaux, J.W.; et al. Is Structured Observation a Valid Technique to Measure Handwashing Behavior? Use of acceleration sensors embedded in soap to assess reactivity to structured observation. American J. Trop. Med. Hyg. 2010, 83, 1070-1076. [CrossRef] [PubMed]

9. Cousens, S.; Kanki, B.; Toure, S.; Diallo, I.; Curtis, V. Reactivity and repeatability of hygiene behaviour: Structured observations from burkina faso. Social Sci. Med. 1996, 43, 1299-1308. [CrossRef]

10. Amitrano, D.; Martino, G.D.; Iodice, A.; Mitidieri, F.; Papa, M.N.; Riccio, D.; Ruello, G. Sentinel-1 for monitoring reservoirs: A performance analysis. Remote Sens. 2014, 6, 10676-10693. [CrossRef]

11. Sobrino, J.; Jiménez-Muñoz, J.C.; Paolini, L. Land surface temperature retrieval from LANDSAT TM 5. Remote Sens. Environ. 2004, 90, 434-440. [CrossRef]

12. Weng, Q.; Lu, D.; Schubring, J. Estimation of land surface temperature-Vegetation abundance relationship for urban heat island studies. Remote Sens. Environ. 2004, 89, 467-483. [CrossRef]

13. Available online: https:/ / www.servirglobal.net (accessed on 15 April 2018).

14. García, L.; Rodríguez, J.D.; Wijnen, M.; Pakulski, I. Earth Observation for Water Resources Management: Current Use and Future Opportunities for the Water Sector; World Bank: Washington, DC, USA, 2016.

15. Available online: http://www.fews.net/content/using-crowdsourcing-map-displacement-south-sudan (accessed on 15 April 2018).

16. World Bank. Nigeria WASH Poverty Diagnostic: Intermediate Review Meeting; World Bank Group: Washington, DC, USA, 2017.

17. World Bank. Predicting Deprivations in Housing and Basic Services from Space; World Bank Group: Washington, DC, USA, 2018.

18. Available online: http://hydrobidlac.org/ (accessed on 15 April 2018).

19. Harwin, S.; Lucieer, A. Assessing the accuracy of georeferenced point clouds produced via multi-view stereopsis from unmanned aerial vehicle (UAV) imagery. Remote Sens. 2012, 4, 1573-1599. [CrossRef]

20. Horcher, A.; Visser, R.J.M. Unmanned aerial vehicles: Applications for natural resource management and monitoring. In Proceedings of the 2004 Council on Forest Engineering (COFE) Conference "Machines and People, The Interface", Hot Springs, AR, USA, 27-30 April 2004.

21. Laliberte, A.; Herrick, J.E.; Rango, A.; Winters, C. Acquisition, orthorectification, and object-based classification of unmanned aerial vehicle (UAV) imagery for rangeland monitoring. Photogramm. Eng. Remote Sens. 2010, 76, 661-672. [CrossRef]

22. Dufour, S.; Bernez, I.; Betbeder, J.; Corgne, S.; Hubert-Moy, L.; Nabucet, J.; Rapinel, S.; Sawtschuk, J.; Trollè, C. Monitoring restored riparian vegetation: How can recent developments in remote sensing sciences help? Knowl. Manag. Aquat. Ecosyst. 2013, 410, 1-15. [CrossRef]

23. Koh, L.P.; Wich, S.A. Dawn of drone ecology: Low-cost autonomous aerial vehicles for conservation. Trop. Conserv. Sci. 2012, 5, 121-132. [CrossRef]

24. Fritz, A.; Kattenborn, T.; Koch, B. UAV-based photogrammetric point clouds-Tree stem mapping in open stands in comparison to terrestrial laser scanner point clouds. Int. Arch. Photogramm. Remote Sens. Spat. Inf. Sci. 2013, XL-1/W2, 141-146. [CrossRef]

25. Zarco-Tejada, P.J.; Diaz-Varela, R.; Angileri, V.; Loudjani, P. Tree height quantification using very high resolution imagery acquired from an unmanned aerial vehicle (UAV) and automatic 3D photo-reconstruction methods. Eur. J. Agron. 2014, 55, 89-99. [CrossRef]

26. Dandois, J.P.; Ellis, E.C. High spatial resolution three-dimensional mapping of vegetation spectral dynamics using computer vision. Remote Sens. Environ. 2013, 136, 259-276. [CrossRef] 
27. Salamí, E.; Barrado, C.; Pastor, E. UAV flight experiments applied to the remote sensing of vegetated areas. Remote Sens. 2014, 6, 11051-11081. [CrossRef]

28. Available online: https:/ / akvo.org (accessed on 15 April 2018).

29. Baker, A.; Cumberland, S.A.; Bradley, C.; Buckley, C.; Bridgeman, J. To what extent can portable fluorescence spectroscopy be used in the real-time assessment of microbial water quality? Sci. Total Environ. 2015, 532, 14-19. [CrossRef] [PubMed]

30. Sorensen, J.P.; Lapworth, D.J.; Marchant, B.P.; Nkhuwa, D.C.; Pedley, S.; Stuart, M.E.; Bell, R.A.; Chirwa, M.; Kabika, J.; Liemisa, M.; et al. In-Situ tryptophan-like fluorescence: A real-time indicator of faecal contamination in drinking water supplies. Water Res. 2015, 81, 38-46. [CrossRef] [PubMed]

31. Coble, P.G.; Lead, J.; Baker, A.; Reynolds, D.M.; Spencer, R.G.M. Aquatic Organic Matter Fluorescence; Cambridge University Press: New York, NY, USA, 2014.

32. United Nations Children's Fund (UNICEF). Real Time E. coli Detection; UNICEF: New York, NY, USA, 2014.

33. Emerson Automated Solutions. Fluoride Monitoring System; Emerson Automated Solutions: Shakopee, MN, USA, 2017.

34. Available online: https://www.analyticaltechnology.com/analyticaltechnology/gas-water-monitors/ product.aspx?ProductID=1052 (accessed on 15 April 2018).

35. Available online: http://www.arsenicbiosensor.org (accessed on 15 April 2018).

36. Available online: http://www.palintest.com/en/products/digital-arsenic-test-kit (accessed on 15 April 2018).

37. Available online: https://www.ipi-singapore.org/technology-offers/digital-arsenic-detector (accessed on 15 April 2018).

38. McAllister, L. We're Sensing a Change in Water Monitoring: Introducing the Arsenic Sensor Prize Competition. Available online: https://blog.epa.gov/blog/2016/09/were-sensing-a-change-in-watermonitoring-introducing-the-arsenic-sensor-prize-competition/ (accessed on 15 April 2018).

39. Available online: https:/ / nextdrop.co (accessed on 15 April 2018).

40. Available online: http://www.sarvajal.com (accessed on 15 April 2018).

41. Koehler, J.; Thomson, P.; Hope, R. Pump-priming payments for sustainable water services in rural Africa. World Dev. 2015, 74, 397-411. [CrossRef]

42. Arniella, E.F. Evaluation of Smart Water Infrastructure Technologies (SWIT); Inter-American Development Bank: Washington, DC, USA, 2016.

43. USAID. USAID Launches 500 Million Birr Activity on World Water Day to Boost Ethiopia's One Wash National Program; USAID: Washington, DC, USA, 2016.

44. U.S. Agency for International Development (USAID). Kenya Resilient Arid Lands Partnership for Integrated Development; USAID: Washington, DC, USA, 2015.

45. Thomas, E.A.; Mattson, K. Instrumented Monitoring with Traditional Public Health Evaluation Methods: An Application to a Water, Sanitation and Hygiene Program in Jakarta, Indonesia; Mercy Corps: Portland, OR, USA, 2013.

46. Thomas, E.A.; Tellez-Sanchez, S.; Wich, C.; Kirby, M.; Zambrano, L.; Rosa, G.; Clasen, T.; Nagel, C. Behavioral reactivity associated with electronic monitoring of environmental health interventions-A cluster randomized trial with water filters and cookstoves. Environ. Sci. Technol. 2016, 50, 3773-3780. [CrossRef] [PubMed]

47. Delea, M.G.; Nagel, C.L.; Thomas, E.A.; Halder, A.K.; Amin, N.; Shoab, A.K.; Freeman, M.C.; Unicomb, L.; Clasen, T.F. Comparison of respondent-reported and sensor-recorded latrine utilization measures in rural Bangladesh: A cross-sectional study. Trans. R. Soc. Trop. Med. Hyg. 2017, 111, 308-315. [CrossRef] [PubMed]

48. Sinha, A.; Nagel, C.L.; Thomas, E.; Schmidt, W.P.; Torondel, B.; Boisson, S.; Clasen, T.F. Assessing latrine use in rural India: A cross-sectional study comparing reported use and passive latrine use monitors. Am. J. Trop. Med. Hyg. 2016, 95, 720-727. [CrossRef] [PubMed]

49. Clasen, T.; Fabini, D.; Boisson, S.; Taneja, J.; Song, J.; Aichinger, E.; Bui, A.; Dadashi, S.; Schmidt, W.P.; Burt, Z.; et al. Making sanitation count: Developing and testing a device for assessing latrine use in low-income settings. Environ. Sci. Technol. 2012, 46, 3295-3303. [CrossRef] [PubMed]

50. Biran, A.; Rabie, T.; Schmidt, W.; Juvekar, S.; Hirve, S.; Curtis, V. Comparing the performance of indicators of hand-washing practices in rural Indian households. Trop. Med. Int. Health 2008, 13, 278-285. [CrossRef] [PubMed] 
51. Sullivan, M. Turning Citizens into Sensors: Using Mobile Apps and "Big Data" to Combat Water Losses in South Africa. Water \& Sanitation for the Urban Poor (blog). Available online: http:/ / www.wsup.com/2015/06/03/turning-citizens-into-sensors-using-mobile-apps-and-bigdata-to-combat-water-losses-in-south-africa/ (accessed on 15 April 2018).

52. World Bank-UN. Using Big Data for the Sustainable Development Goals. Available online: https://unstats.un.org/unsd/trade/events/2015/abudhabi/presentations/day3/02/2b\%20A-Using\% 20Big\%20Data\%20for\%20the\%20Sustainable\%20Development\%20Goals\%2010222015.pdf (accessed on 15 April 2018).

53. Thomas, E.A.; Andres, L.A.; Borja-Vega, C.; Sturzenegger, S. Innovations in WASH Impact Measures: Water and Sanitation Measurement Technologies and Practices to Inform the Sustainable Development Goals. In Directions in Development-Infrastructure; World Bank: Washington, DC, USA, 2018.

2018 by the authors. Licensee MDPI, Basel, Switzerland. This article is an open access article distributed under the terms and conditions of the Creative Commons Attribution (CC BY) license (http:/ / creativecommons.org/licenses/by/4.0/). 